\title{
Electrical FIR Filter With Optical Coefficients for Self-Referencing WDM Intensity Sensors
}

\author{
J. Montalvo, F. M. Araújo, L. A. Ferreira, C. Vázquez, Senior Member, IEEE, and \\ J. M. Baptista, Senior Member, IEEE
}

\begin{abstract}
In this letter, a novel radio-frequency finite impulse reponse (FIR) electrooptical configuration for self-referencing optical wavelength-division-multiplexed intensity sensors is reported. By employing the proposed low-cost electronic FIR filter at the reception stage, the use of a 14-km fiber delay coil is avoided for a $2-\mathbf{k H z}$ modulation frequency, preserving sensitivity and self-reference. The technique is analyzed following the Z-transform formalism and measurements validating the theoretical model are reported.
\end{abstract}

Index Terms-Fiber Bragg gratings (FBGs), fiber-optic sensors, finite impulse response (FIR), optical filters, self-reference, wavelength-division multiplexing (WDM).

\section{INTRODUCTION}

O PTICAL signal processing theory is widely developed and configurations for optical sensors and communications networks are constantly being deployed.

First, all-optical incoherent signal processors based on fiberoptic technology [1] were reported in order to overcome the bandwidth constraints of microwave and radio-frequency (RF) signal filters. In these photonic devices, the time delays in the optical paths are much higher than the coherence time of the employed optical sources and the coefficients of the filters are always positive.

On the other hand, when the time delays in the optical paths are comparable to the coherence time of the optical sources, the optical phase relations between the different ports are deterministic, and coherent filters are achieved. Several photonic synthesis techniques based on transversal filters [2], delay line lattices [3], and ring resonators [4], [5] were reported in the last decade. This kind of filter provides greater design flexibility and higher processing bandwidths up to several terahertz.

Manuscript received July 31, 2007; revised September 24, 2007. This work was supported by COST Action 299-Optical Fibres for New Challenges Facing the Information Society "FIDES," Spanish CICYT TEC2006-13273-C03-03-MIC and Mobility Action 2007 (University Carlos III of Madrid).

J. Montalvo and C. Vázquez are with the Department de Tecnología Electrónica, Universidad Carlos III de Madrid, Leganés, 28911 Madrid, Spain (e-mail: julio.montalvo@uc3m.es).

F. M. Araújo and L. A. Ferreira are with INESC Porto, Unidade de Optoelectrónica e Sistemas Electrónicos, 4169-007 Porto, Portugal.

J. M. Baptista is with INESC Porto, Unidade de Optoelectrónica e Sistemas Electrónicos, 4169-007 Porto, Portugal, and also with the Department de Matemática e Engenharias, Universidade da Madeira, 9000-390 Funchal, Portugal (e-mail: jmb@inescporto.pt).

Color versions of one or more of the figures in this letter are available online at http://ieeexplore.ieee.org.

Digital Object Identifier 10.1109/LPT.2007.911511
Coherent filters are used to achieve high-speed components for communication systems such as switches, modulators, and tunable filters for dense wavelength-division-multiplexing (WDM) networks with ultrahigh data traffic [6].

In the case of sensor networks, the amount of information to be addressed is usually very low and quasi-static, being low-cost devices, efficient multiplexing topologies [7], and self-referenced measurements [8] relevant aspects. In this environment, incoherent fiber-optic configurations with wavelength multiplexing and reflective operation are the most desirable approaches.

Different RF self-referencing techniques for intensity sensors based on incoherent Sagnac [9] and Michelson [10] interferometers, fiber delay lines [11], and ring resonators [12] have been reported. In all of them, the optical power modulation of the sensor at the sensing point is related to the coefficients of the filter structure, thus encoding the shape of the filter response either in magnitude or in phase and performing self-referenced measurements. Unfortunately, in order to allow operating RF frequencies in the order of $1 \mathrm{kHz}$, the length of the required delay fiber coils is in the order of $10 \mathrm{~km}$, due to the fact that the light propagation time in the fiber is used to achieve a RF phase-shift between the electrical beating signals in the photodetector.

In this letter, a novel RF electrooptical finite impulse reponse (FIR) configuration for self-referencing fiber-optic intensity sensors is reported. It is based on the configuration reported in [11], but the long fiber delay coil in each measuring point is replaced by a compact and reconfigurable electronic delay in the processing unit [see Fig. 1(a)].

\section{THEORY}

The proposed fiber-optic configuration with a remote intensity sensor placed within two fiber Bragg gratings (FBGs) and the electrical reception stage is shown in Fig. 1(a). A broadband light source (BLS) is modulated at a fixed RF $f$. The optical signal is sliced in wavelength and reflected by two FBGs at the remote sensing point. The central wavelengths of the FBGs placed before and after the optical sensor (fiber-optic sensor) are named $\lambda_{R}$ (reference) and $\lambda_{S}$ (sensor), respectively. The sensor power modulation is named $H$. In the reception stage, an electrical phase-shift $\Omega_{d}$ is applied to the RF modulating signal at the reflected sensor wavelength $\lambda_{S}$. Then a delay line filter is deployed in the electrical domain but with a coefficient $(\beta)$, which depends on the optical power modulation $H$ in the sensing point.

The FIR filter schematic of the complete sensor topology is shown in Fig. 1(b). Identifying a generic electrical phase-shift $(\Omega)$ with the characteristic transit time of an equivalent digital 


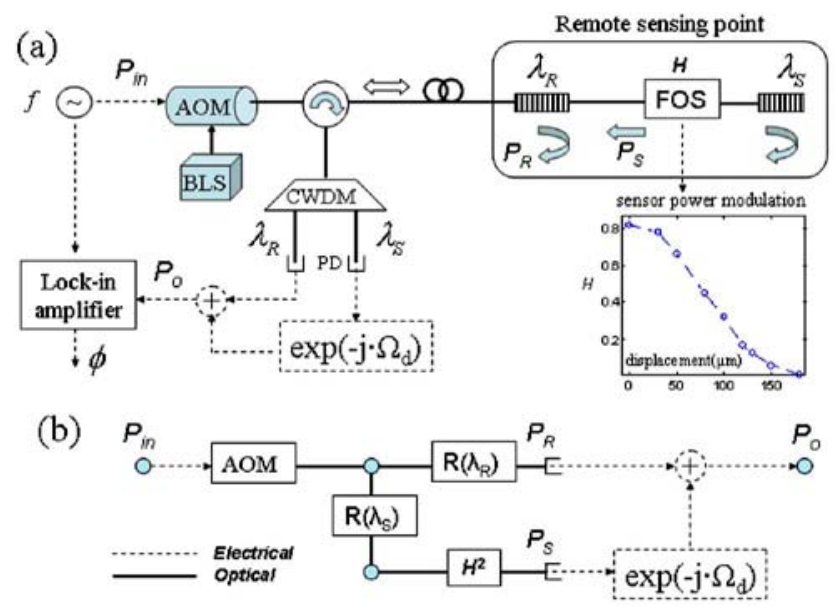

Fig. 1. (a) Electrooptical configuration and (b) filter model for the proposed self-referencing technique without delay fiber coil. AOM: acoustooptic modulator; FOS: fiber-optic sensor; PD: photodetector.

filter $\left(\exp (-j \cdot \Omega)=z^{-1}\right)$, the transfer function can be expressed in the Z-transform domain as follows:

$$
\frac{P_{o}}{P_{\text {in }}}=\alpha \cdot\left(1+\beta \cdot z^{-1}\right)
$$

where

$$
\begin{aligned}
& \alpha=m_{r} \cdot R\left(\lambda_{R}\right) \cdot d_{r} \\
& \beta=\frac{m_{s} \cdot R\left(\lambda_{S}\right) \cdot d_{s}}{m_{r} \cdot R\left(\lambda_{R}\right) \cdot d_{r}} \cdot H^{2}
\end{aligned}
$$

where $m_{r} \cdot R\left(\lambda_{R}\right) \cdot d_{r}$ is the RF modulation index, the reflectivity of the FBG, and the photodetector response at the reference wavelength $\lambda_{R}$, respectively, and $m_{s} \cdot R\left(\lambda_{S}\right) \cdot d_{s}$ is the respective similar parameters for the sensor wavelength $\lambda_{S}$.

The sensor power modulation $H$, which depends on the measurand, is remotely addressed through the optical link and is encoded in the transfer function of the self-referencing configuration by means of the parameter $\beta$.

The electrical phase response of the detected RF signal, namely $\phi$, has the following expression:

$$
\phi=\arctan \left(\frac{-\beta \cdot \sin \Omega}{1+\beta \cdot \cos \Omega}\right)
$$

where $\Omega$ is the angular frequency.

Equation (4) shows that $\phi$ is insensitive to power fluctuations except for the sensor modulation $H$, thus performing as a selfreference measurement parameter. The phase response versus angular frequency $\Omega$ of the FIR filter of Fig. 1(b) is shown in Fig. 2(b) for different values of $\beta$. An anti-symmetrical phase shape can be seen with respect to $\Omega=\pi$.

The proposed configuration avoids the need for a fiber delay coil between the two FBGs at the sensing point. Note that a restrictive tradeoff between $f$ and the length $L$ of the delay fiber takes place in the all-optical self-referencing approach [11] (a) Normalized magnitude response

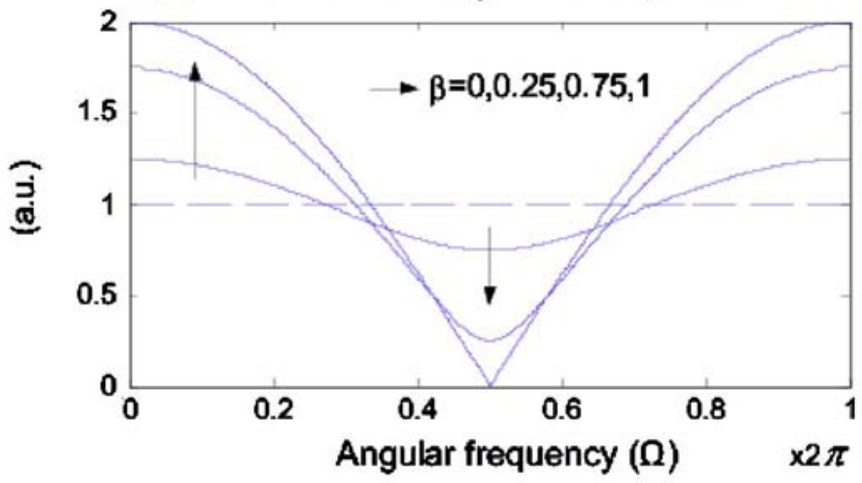

(b)

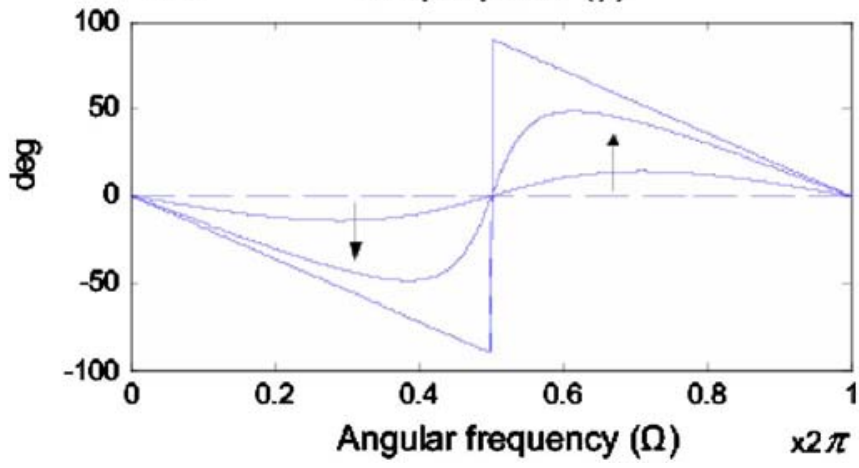

Fig. 2. Transfer function of the self-referencing configuration versus angular frequency $(\Omega)$ for different values of $\beta$. Dashed lines: $\beta=0$.

$\left(\Omega_{d}=(2 \pi / c) \cdot n_{g} \cdot f \cdot 2 L\right.$, where $n_{g}$ is the effective group refractive index of optical fiber and $c$ is the speed of light). With the electrical FIR filter, this tradeoff is avoided and a more compact, efficient, and flexible configuration is achieved, at the expense of using an additional wavelength demultiplexer and photodetector.

\section{MEASUREMENTS}

The electrooptical configuration shown in Fig. 1(a) has been implemented using single-mode fiber with FC/APC connectors. An erbium-doped BLS modulated at $f=2 \mathrm{kHz}$ by an acoustooptic modulator was employed to launch optical power within the $C$-band (1510-1560 nm) into the configuration. Two FBGs with a $3-\mathrm{dB}$ bandwidth of $0.25 \mathrm{~nm}$ have been used in the remote sensing point. Their central wavelengths are $\lambda_{R}=1536 \mathrm{~nm}$ and $\lambda_{S}=1552.5 \mathrm{~nm}$, and their reflectivities are $43 \%$ and $48 \%$, respectively. A single-mode fiber taper operating as a microdisplacement sensor is placed between the two FBGs and used to calibrate the configuration [see the inset of Fig. 1(a)].

The reflected waves are demultiplexed by a coarse WDM (CWDM) and collected by two distinct InGaAs detectors. The electronic delay line filter shown in Fig. 1(b) is then implemented using commercial capacitors, variable resistors, and LM741 operational amplifiers. Finally, the output $P_{o}$ is connected to a lock-in amplifier to obtain the output phase $(\phi)$ as the self-referenced parameter.

Provided that the lock-in amplifier is locked to the phase of the reflected reference wavelength (at $\beta=0$ ), the acquired 


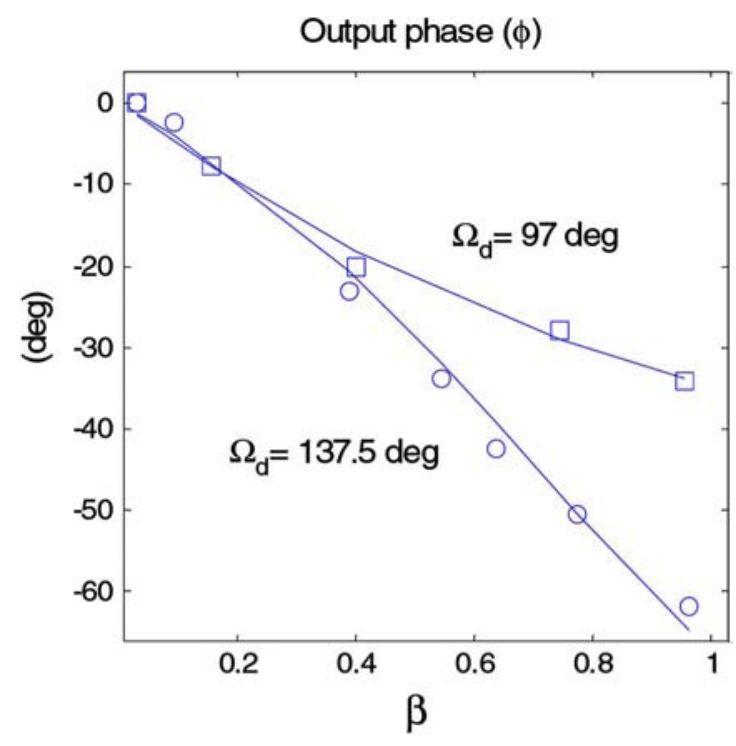

Fig. 3. Self-referenced parameter measurements versus $\beta$ for different electrical phase-shifting values. Theoretical curves are drawn in continuous lines.

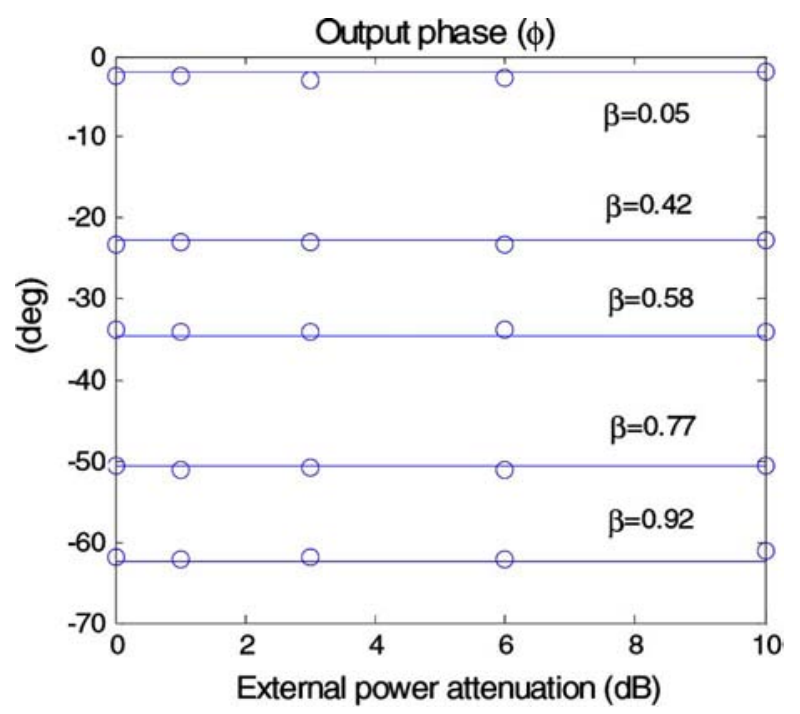

Fig. 4. Sensitivity of the self-referenced parameter to external attenuation for $\Omega_{d}=137.5^{\circ}$ and different values of $\beta$. Theoretical curves are drawn in continuous lines.

output phase and the induced phase-shift $\Omega_{d}$ can be identified with $\phi$ and $\Omega$ of Fig. 2, respectively. So the phase-shifting circuit determines the operating point of the configuration in a very simple and flexible way.

Different calibration curves versus $\beta$ have been obtained for different phase-shifting values, showing good agreement between theory and measurements (see Fig. 3). Note that a fiber delay coil with a length of $300 \mathrm{~m}$ is needed to fix a phase-shift around $100^{\circ}$ for a modulating frequency of $100 \mathrm{kHz}$ in [11], while in this case no fiber coil is required, a higher phase-shift is achieved and the modulating frequency is 50 times lower.

A variable optical attenuator has been used for inducing power fluctuation in the RF modulated optical source to test the self-reference of the measurements. No change in the self-referenced output phase has been noticed after inducing
$10 \mathrm{~dB}$ of power attenuation (see Fig. 4). A minimum power density of $0.73 \mu \mathrm{W} / \mathrm{nm}$ is required at the BLS for proper operation.

By using a BLS with a spectrum within the 1270- to 1610-nm band, we estimate that a low-cost CWDM sensor network with up to 16 intensity sensors could be achieved, using two FBGs for each sensor with central wavelengths in the same CWDM channel.

\section{CONCLUSION}

A novel RF electrooptical FIR configuration for self-referencing WDM fiber-optic intensity sensors is reported. It provides enhanced flexibility, compact design, and single point reconfiguration of multiple sensors. Long fiber delay coils of hundreds of meters are avoided and the operating point of the topology can be adjusted just by fixing an electrical phase-shift of an RF signal at $2 \mathrm{kHz}$ in a simple and flexible way. This frequency is arbitrary so other values can be used if it is required for integrating the configuration in any other electronic application. The technique can be applied to FIR optical configurations and is compatible with wavelength multiplexing of intensity sensors in optical networks.

\section{ACKNOWLEDGMENT}

The authors would like to thank J. L. Santos and O. Frazão for their helpful suggestions and assistance in the experiments.

\section{REFERENCES}

[1] K. P. Jackson, S. A. Newton, B. Moslehi, M. Tur, C. C. Cutler, J. W. Goodman, and H. J. Shaw, "Optical fiber delay-line signal processing," IEEE Trans. Microw. Theory Tech., vol. MTT-33, no. 3, pp. 193-210, Mar. 1985

[2] O. K. Sasayama, M. Okuno, and K. Habara, "Coherent optical transversal filter using silica-based waveguides for high-speed signal processing," J. Lightw. Technol., vol. 9, no. 10, pp. 1225-1230, Oct. 1991.

[3] K. Jinguji and M. Kawachi, "Synthesis of coherent two-port latticeform optical delay-line circuit," J. Lightw. Technol., vol. 13, no. 1, pp. 73-82, Jan. 1995.

[4] R. Orta, P. Savi, R. Tascone, and D. Trinchero, "Synthesis of multiplering-resonator filters for optical systems," IEEE Photon. Technol. Lett., vol. 7, no. 12, pp. 1447-1449, Dec. 1995.

[5] A. Melloni, "Parallel-coupled ring-resonators filters synthesis," Opt. Lett., vol. 26, no. 12, pp. 917-919, Jun. 2001.

[6] V. R. Almeida, C. A. Barrios, R. R. Panepucci, and M. Lipson, "Alloptical control of light on a silicon chip," Lett. Nature, vol. 431, pp. 1081-1083, 2004.

[7] J. Montalvo, C. Vázquez, and D. S. Montero, "CWDM self-referencing sensor network based on ring resonators in reflective configuration," Opt. Express, vol. 14, no. 11, pp. 4601-4610, 2006.

[8] C. Vázquez, J. Montalvo, and P. C. Lallana, "Radio-frequency ring resonators for self-referencing fiber-optic intensity sensors," Opt. Eng. Lett., vol. 44, pp. 1-2, 2005

[9] J. M. Baptista, J. L. Santos, and A. S. Lage, "Self-referenced fibre optic intensity sensor based on a multiple beam Sagnac topology," Opt. Commun., vol. 181, pp. 287-294, 2000.

[10] J. M. Baptista, S. Abad, G. M. Rego, L. A. Ferreira, and J. L. Santos, "Wavelength multiplexing of frequency-based self-referenced fiber optic intensity sensors," Opt. Eng., vol. 43, no. 3, pp. 702-707, 2004.

[11] S. Abad, M. López-Amo, F. M. Araújo, L. A. Ferreira, and J. L. Santos, "Fiber Bragg grating-based self-referencing technique for wavelengthmultiplexed intensity sensors," Opt. Lett., vol. 27, pp. 222-224, 2002.

[12] C. Vázquez, J. Montalvo, D. S. Montero, and J. M. S. Pena, "Self-Referencing fiber-optic intensity sensors using ring resonators and fiber Bragg gratings," IEEE Photon. Technol. Lett., vol. 18, no. 22, pp. 2374-2376, Nov. 15, 2006. 